\title{
Artificial Intelligence and the Problem of Computer Representation of Knowledge*
}

\author{
Vladimir Inozemtsev \\ National Research University \\ Moscow State Technical University named after N.E. Bauman \\ (MSTU named after N. E. Bauman) \\ 5/1 2d Baumanskaya Street, Moscow, Russia 105005 \\ E-mail: inozem_63@mail.ru
}

\author{
Marina Ivleva \\ Faculty of Humanities and Social Sciences \\ Department of Social Philosophy \\ Peoples' Friendship University of Russia \\ (RUDN University) \\ 6 Miklukho-Maklaya Street, Moscow, 117198, Russia \\ E-mail: marinanonna@yandex.ru
}

\author{
Vitaly Ivlev \\ National Research University \\ Moscow State Technical University named after N.E. \\ Bauman \\ (MSTU named after N.E.Bauman) \\ 5/1 2d Baumanskaya Street, Moscow, 105005, Russia \\ E-mail: vitalijivlev@yandex.ru
}

\begin{abstract}
The article developed the concept of epistemology of artificial intelligence (AI-epistemology), the concept of computer epistemology and the concept of computer representology. In this work, the understanding of artificial intelligence as a project, the theory and the totality of specific applications (so-called intellectual systems) differs; the analysis of the most important problem of artificial intelligence - the problem of computer representation of knowledge; the main stages of the development of the theory of artificial intelligence - classical, nonclassical and post-nonclassical - are analyzed and compared with the corresponding types of scientific rationality.
\end{abstract}

Keywords-Problem of computer representation of knowledge; cognitive sciences; computer sciences; artificial intelligence (AI); epistemology of artificial intelligence; computer epistemology; computer representology

\section{INTRODUCTION}

The concept of epistemology of artificial intelligence refers to the analysis of new epistemological problems, including problems of representation, manipulation, acquisition, replenishment, generalization and classification of computer knowledge, problems of computer perception and computer understanding.

Epistemology of artificial intelligence (AI-epistemology) is a section of computer epistemology [1]. In computer epistemology, the problems of understanding the essence of information are investigated; studying the mechanisms of

This paper was financially supported by the Ministry of Education and Science of the Russian Federation on the program to improve the competitiveness of Peoples' Friendship University (RUDN University) among the world's leading research and education centers in the 2016-2020 (The Agreement number 02.A03.21.0008). storage, translation and acquisition of information in computer systems (including computer knowledge); analysis of concepts of knowledge engineering; and a number of others. In computer epistemology, the origin, development and specificity of computer data and computer knowledge are studied; the formation of computer knowledge from computer data.

In the concept of computer representology, an analysis of the specifics, formation and development, as well as the most important ways to solve the problem of computer representation of knowledge. These various ways of solving this problem are embodied in the creation of three leading concepts of computer representation of knowledge (logical, network, frame), each of which is a collection of relevant models and languages of computer representation of knowledge.

\section{THE HISTORY OF RESEARCH IN THE FIELD OF ARTIFICIAL INTELLIGENCE}

In the middle of the $\mathrm{XX}$ century, as a result of using computers to solve problems of symbolic information processing, an interdisciplinary direction of research appears, that is called "artificial intelligence" (AI) [2, 3]. It should be noted that the term "AI" is introduced into the scientific lexicon in 1956 by J. McCarthy. Researches in the field of AI become in the mid-1950s the vanguard of the so-called cybernetic movement, which is initiated by $\mathrm{N}$. Wiener.

The cybernetic movement in the 1940s-1960s serves as a single theoretical and applied base for a number of scientific disciplines that study information processing processes, as well as control and communication problems in complex, dynamic, computing systems [4]. It is thanks to cybernetics 
that an idea is formed about the similarity of patterns that determine the processes of processing information and control in computer systems and in humans [5]. This idea is also joined by the idea of the fruitfulness of analyzing these processes with the help of logical-linguistic models [6], which form a special kind of conceptual sign models, which later find wide application in AI research, especially in the computer representation of knowledge. The final recognition of the term "artificial intelligence" occurs almost a decade and a half after its appearance, in 1969, when the first international conjoint conference on artificial intelligence was held.

\section{THE CONCEPT OF EPISTEMOLOGY OF ARTIFICIAL INTELLIGENCE}

The improvement of intelligent information technologies in recent decades necessitated a new outlook at natural intelligence and the possibility of its computer simulation [7, 8 ]. There is a range of issues and ideas relating to this new section of epistemology that could be called AIepistemology (epistemology of artificial intelligence). In AI epistemology, there are set and solved the problems of the relation of human and machine intelligence; $\mathrm{AI}$; computer perception and computer understanding; problems of representation, acquisition, processing, transformation, and replenishment of computer knowledge; analysis of their specificity, the structural organization and functioning. The efforts of classical academic epistemology that originated in the early XVII century and finalized by the beginning of the twentieth century are not enough to solve these problems anymore. Despite the fact that by this time the orbit of epistemological survey includes the problems of technical knowledge, research, technologies, specifics of engineering thinking, but there are no major transformations during this period in the subject matter of epistemology, its methods and objectives. In this work, we study only part of the problems related to the above mentioned problems of AI epistemology, and it is those that are directly relevant to the epistemological content of AI.

The era of change starts in the 50 s - 60s years of the twentieth century and is associated with the development of information technologies and, in particular, intelligent technology that gives impetus to the formation of computer epistemology and AI epistemology as sections of postnonclassical epistemology. During this period, researchers refer to the solution of the problems of philosophical understanding of information and intelligence [9]; the specifics of the computer knowledge that grow out of computer data in the process of development of information and communication technologies. Epistemology of artificial intelligence studies in computer knowledge from the standpoint of representation, transformation and transmission of information, and turning ordinary knowledge into computer knowledge. This epistemology focuses on the analysis of the new epistemological problems. In developing the variant AI-epistemology investigates the problem of representation, manipulation, acquisition, replenishment, consolidation and classification of computer knowledge and problems of computer perception and computer understanding.

In this research the interest of AI epistemology is shifting towards everyday knowledge and computer representation of the results of this form of knowledge (although, of course, not only). This is due to the fact that ordinary knowledge is the source and basic form of cognitive activity. This form is universal, complex and versatile, which makes it possible to understand how to transform normal information in the computer knowledge. Intellectual activity in AI epistemology is understood as the implementation of certain cognitive structures and processes, and this brings it closer to that section of representology as cognitive representology. Cognitive processes in the new epistemology are understood as a process of transformation of computer information, and thinking is defined as a computational process. In this regard, AI epistemologies arises and develop new ideas, approaches and concepts that are different from classical epistemology. AI computer epistemology considers knowledge as the highest form of representation of information in the AI. On this basis the goals, objectives and accents characteristic of classical epistemology, are radically changed in the epistemology of artificial intelligence. However, AI epistemology and classical epistemology have some area of intersection.

\section{THE CONCEPT OF COMPUTER EPISTEMOLOGY}

AI epistemology is one of the most important sections of computer epistemology. In computer epistemology, which is broader than AI epistemology, the direction of postnonclassical (postclassical) epistemology, there is an appeal to the study of a range of problems, and methods are used to solve these problems that are not characteristic of classical epistemology. Among the problems of computer epistemology, along with the above problems of AI epistemology, also include the problems of studying the mechanisms of storage, translation and acquisition of information in computer systems (including computer knowledge); research of formation and development of two forms of information representation in computer sciences and AI - computer data and computer knowledge; and also the formation of computer knowledge from computer data. The presence of new problems and the development of ways to solve these problems turn computer epistemology into a new, independent section of modern post-nonclassical epistemology. Understanding of computer epistemology is a fairly close understanding of information epistemology, developed in the work of Rakitov "The Philosophy of the Computer Revolution" [10].

Over the past decades, from the very appearance of the very term "AI" and up to the present day, there is no such generally accepted definition of this term suiting all specialists engaged in research in this field. Usually "AI" means a combination of software and hardware, the application of which leads to results similar to those obtained through the implementation of human intellectual activity. At the same time, it can be argued that any of the methods of rational cognition, involving the construction of a picture of the world in accordance with certain rules, is essentially the 
creation of some models of knowledge in AI [11], or more precisely, models of representation of knowledge in AI.

\section{THE BASIC APPROACHES TO UNDERSTANDING ARTIFICIAL INTELLIGENCE}

We will distinguish $\mathrm{AI}$ as a project, theory and a set of specific applications (the so-called intelligent information systems). The AI project involves the technical embodiment of the old dream of many thinkers and scientists - the modeling of people's intellectual abilities or computer (computer) modeling of thinking. To implement this project in the late 1950s - early 1960s, the development of the theory of AI has began, which should be understood as a set of software and hardware capable of realizing the intellectual activity of computers that is comparable to human intellectual activity.

The AI theory includes a number of directions and sections of AI research, which are a solution to the relevant problems of AI. Such areas and sections of AI include: problem solving and proof of theorems, representation and manipulation of knowledge, training, computer perception and computer understanding, engineering (acquisition) of knowledge and planning of expedient activities, and a number of others. Like any science, AI theory has its own object and methods of investigation. The specific object of research in $\mathrm{AI}$ is met procedures that mimic the intellectual activity of people, and the process of implementing these met procedures in technical systems. As methods of research in AI, a lot of deductive, empirical and descriptive methods are used. Finally, specific AI applications are intellectual systems created with the help of intelligent information technology tools and acting as a practical implementation in the form of various expert and other intellectual systems.

From the very beginning of implementation of AI project, various science specialists took part in it: programmers, psychologists, mathematicians, philosophers, linguists, logicians, biologists [12]. This is the first time in the history of science such a large-scale interdisciplinary research is being undertaken. The most important purpose of AI, according to the plan of its creators, should be computer modeling of thinking.

Just a decade after the beginning of development in AI, two competing basic paradigms of computer modeling of thinking have emerged and still exist, which are called representational and connectionist [13]. In accordance with the first paradigm, the process of thinking is identified with computer processing of symbolic languages, embodying the construction of logical-linguistic models and languages of computer representation of knowledge. Connectionist paradigm identifies computers with the human brain, and the process of thinking - with the dynamics of neural networks. Computers are considered as technical devices that functionally reproduce brain processes on an inorganic substrate.

In recent decades AI supporters have formulated two main positions in understanding AI: the position of "strong AI" and the position of "weak AI". The position of "strong AI" at the present time in the most vivid form is expressed by D. Dennett [14]. His understanding of AI can be characterized by a number of provisions. First, the human consciousness "without a remnant" can fundamentally be reducible to computational models and processes, and fully explain them. Secondly, computers actually "think". Thirdly, computers can be endowed with a spectrum of mental properties that were considered human - pain, desire, sadness. The main condition for the implementation of all these provisions is the proper way to program computers.

Supporters of "weak AI" (J. Searle, T. Vinograd and others) believe that AI acts as a tool for modeling human cognitive abilities, and computers imitate only the cognitive side of thinking $[15,16]$. Understanding and rapport, in their opinion, can not be modeled with computers. "Weak AI" does not pretend either to model thinking in general or to what computers thinks. Computer programs in accordance with this approach are considered intelligent if they pass the Turing test, that is, they reliably imitate the human ability to verbalize the mental activity. In recent decades, various modifications have appeared: the French test, the Watt test.

\section{THE CONCEPT OF COMPUTER REPRESENTOLOGY}

In recent decades, the problem of computer representation of knowledge has come to the forefront of AI research. The essence of the problem of computer representation of knowledge consists in the discrepancy between the experts' unformalized knowledge of the laws of the structure and functioning of subject areas of reality, as well as the informal methods used by experts to solve complex intellectual problems, on the one hand. And, the need to formalize and represent this knowledge in the memory of computers in the form of computer knowledge, on the other hand.

This problem is solved by creating various concepts of computer representation of knowledge (logical, network, frame), each of which is a collection of relevant models and languages of computer representation of knowledge. This problem is a modern technical variant of the more general problem of the representation of knowledge.

The problem of computer representation of knowledge is the core of the epistemological content of AI, which is a set of epistemological problems and methods for solving them, related to transformations in the ways of producing computer knowledge, fixing, processing and using them. The emergence of these problems is caused by the intensive development and application of intellectual and other modern information technologies, as well as reflection over computer knowledge. The AI epistemological content includes the sections of AI research that solve the relevant problems of AI, such as knowledge manipulation, replenishment, generalization and classification, knowledge acquisition, computer perception and computer understanding.

It is necessary to distinguish between the epistemological content of AI in the broad and narrow sense of the word. Under the epistemological content of AI in the narrow sense of the word, we mean the totality of epistemological ideas, problems and methods for their solution, the computer 
generated representation of knowledge and the manipulation of knowledge. And by means of the last term we will denote such section of the AI in which the study of various ways of reasoning and conclusions of some computer knowledge from others is carried out. The epistemological content of AI in the broad sense of the word form epistemological problems and ways of solving them, which are characteristic of the concepts of knowledge engineering, exploring, along with computer representation of knowledge and knowledge manipulation, the problems of acquiring knowledge, replenishing and generalizing them, as well as computer perception and computer understanding.

Attempts at computer modeling of thinking since the inception of this idea give a significant impetus to research in cognitive psychology aimed at studying the cognitive activity of people. Initially, the research in cognitive psychology in the 1950s-1960s were significantly influenced by a computer metaphor that is based on the analogy of people's cognitive activity with computer processing of information. From that point, achievements and successes of computer science and AI constantly influence cognitive science. On the other hand, from the turn of the 1960 s to the 1970s, the results and achievements of cognitive sciences begin to have a reverse effect on $\mathrm{AI}$ and computer representation of knowledge.

Of all the main areas, sections and problems of AI, computer representation of knowledge is most closely associated with philosophy and logic. This relationship is fruitful for both sides. The achievements in $\mathrm{AI}$ in general and in the computer representation of knowledge in particular have an impact on the development of the philosophy of science and technology, the methodology of scientific knowledge, contribute to research in nonclassical (quasicontradictory, pseudophysical, non-monotonic and other) logics. Philosophy and logic, on the other hand, develop logical and methodological tools for AI and computer representation of knowledge. For example, the theory of concepts, model theory, mathematical category theory, $\lambda$ calculus and some other logical theories find wide application for computer representation of knowledge.

Within AI there are a number of problems, as well as sections and areas of research, which are the result of solving relevant problems. The most important sections of AI research are the following sections: problem solving; automatic output; knowledge manipulation; pattern recognition (computer perception); computer processing of natural languages; computer understanding; training in intellectual systems; planning of expedient activities. The problem of computer representation of knowledge is intertwined with almost all these problems and sections of AI. These sections of AI research are based on the results obtained in the course of solving the problem of computer representation of knowledge.

\section{THE STAGES OF THE DEVELOPMENT OF THE THEORY OF ARTIFICIAL INTELLIGENCE}

Let us turn to the philosophical and methodological analysis of the main stages in the development of AI theory in relation to the transformation of types of scientific rationality, while focusing on the typology of scientific rationality proposed by Stepin [17]. In the development of AI theory, a situation similar to the development of other sciences is observed. Differences are associated only with some delay in the passage of the corresponding stages, which is due to the youth of the AI, whose work begins in the mid1950 s and is conceptually formalized by the early 1960s. Let us single out the classical, nonclassical and post-nonclassical stages in the development of the theory of AI, which correspond to the types of scientific rationality dominating at each of these stages [18].

The first stage - the classical one - begins with the era of the cybernetic boom of the early 1950s and ends at the turn of the 1970s-1980s, when the classic paradigm crisis in the AI comes. For the classical period, the paradigm of the socalled "universal" and "autonomous" AI is characteristic, the essence of which is to find a universal means for modeling intellectual processes. At the beginning of the classical period, specialists in the field of AI mainly deal with imitating creative processes on computers, creating game programs, proving theorems, recognizing images, machine translation.

In the second phase of the classics, beginning in the late 1960s, the study of the epistemological content of AI in the broad sense of the word is brought to the forefront. Despite the complexity and variety of approaches to building AI within the classical paradigm of AI, the conceptual principles inherent in all studies of the era of the classical type of scientific rationality are dominant. Among these principles of the classics are the following: 1) fundamentalism; 2) essentialism; 3) dynamic determinism; 4) mechanism; 5) cumulativism; 6) mechanism; 7) analytism; 8) reductionism; 9) naive realism; 10) focus on the establishment and definition of simple elements of complex structures. The classical paradigm of $\mathrm{AI}$, as well as the whole classical type of rationality, is inherent in the epistemological utopia of the necessary, once and for all definite, irrefutable scientific knowledge. AI research in this period is dominated by childish optimism and belief in the speedy implementation of the AI project, within any of the approaches to building AI.

During the dominance of the classical AI paradigm, such a section of AI begins to take shape as a computer representation of knowledge. This section becomes for many years, according to most specialists in AI, the central section of AI research. In the epistemological content of AI in the narrow sense of the word, a classical paradigm is formed, which includes a pair of "classical concepts of computer representation of knowledge + output," where the conclusion is understood to be primarily a reliable conclusion.

If we consider the classical paradigm of AI in the logicalepistemological sense, then it is easy for us to find historical and philosophical analogies in Popper's third world, the absolute idea of Hegel and even the world of Plato's ideas. In each of these cases there are varieties of one and the same subject - the transpersonal, autonomous, objective super mind. The experience of the development of computer science at the turn of the 1970s-1980s leads researchers to 
the conclusion that AI, based on the classical paradigm, is helpless in solving most problems that experts effectively cope with in certain subject areas.

The next stage in the development of the theory of AI is nonclassical - in comparison with other sciences in which it predominates since the 1920 s, it begins relatively late in AI research - in the early 1980s. The nonclassical stage in the development of the theory of AI is directly related to the development of expert systems and the widespread introduction of personal computers. The core of this stage of development of $\mathrm{AI}$ is the paradigm of the so-called "specific" AI.

The principal features of the nonclassical stage, which dominates in AI research in the 1980s and 1990s, are: 1) the activity approach; 2) anti-reductionism; 3) heterogeneity and polymorphism; 4) integralism and polyvariance; 5) relativism; 6) nonlinearity and coherence. In the epistemological content of AI in the narrow sense of the word, the classical paradigm "classical concepts of computer representation of knowledge + withdrawal" replaces the nonclassical paradigm "non-classical concepts of computer representation of knowledge + rationale". During this period, there are discussions of the problem of incompleteness of knowledge and the possibility of their replenishment in AI.

The non-classical paradigm and its implementation in expert systems begin to assert the special role of personal knowledge of specialist experts. This paradigm rehabilitates the role of subjective, implicit knowledge, which, thanks to the efforts of the abstractly impersonal non-subject epistemology, which dominates in philosophy and the classical paradigm of AI, goes back to the epistemology of Hegel, reduces this implicit personal knowledge to the level of a disenfranchised appendage of the transpersonal world of ideas. The ideas of the non-classical AI paradigm are consonant with the ideas of the personal epistemology of Polanyi and the monadology of Leibniz.

The arguments of the supporters of individual, impersonal and individual-personal ways of researching and modeling knowledge in AI are resolved by the beginning and mid-1980s in favor of apologists for personal knowledge. This circumstance is promoted by developments in the field of expert systems. The methodology of an abstract epistemological subject, which had claimed the unconditional domination, both in philosophy and in AI theory, thereby indicates its actual place - the conceptual basis of epistemology, which ascribes only its basics, but is incapable of adequately categorical comprehension of the real cognitive process. The study of subjective, implicit, personal knowledge stands out from this time as a new paradigm of modern Western philosophy of science, within which there is a transition from impersonal epistemology to epistemology, the central point of which is personal knowledge.

The non-classical paradigm of AI marks a turn to computer modeling of personal knowledge of experts competent in various subject areas. This paradigm does not focus on objectified, strictly formalized knowledge, as in the case of the classical paradigm, but on incomplete, inaccurate, implicit, personal, subjective, sometimes even contradictory, poorly formalized expert knowledge. The transition from the modeling of knowledge of subject domains to the modeling of expertise acquires enormous importance in recent decades - expert systems become the basis of modern information and computer civilization. The commonality of the subject of research (patterns of representation and transformation of knowledge in people and computers) contributes to the fact that within the framework of the nonclassical paradigm, occurs interpenetration, mutual enrichment and mutual development of cognitive and computer sciences.

From the mid-1980s, a new field of theoretical and practical activity is developing intensively to identify expert knowledge and their representation in computers knowledge engineering in the narrow sense of the word, new cognitive sciences appear that explore the personal form of being knowledge - the psychology of knowledge, cognitive psycholinguistics, psychosemantics and others. All this leads to the realization in the last two decades of the XX century of the finiteness of the individual-impersonal non-subject epistemology that was dominant until then. During this period, there is a transition from abstract epistemological models of knowledge and models of representation of knowledge based on this epistemology to new subjectoriented models that represent personal, individual knowledge in their categorical schemes.

It is within the framework of the nonclassical paradigm of AI that a need for the construction of a new epistemological concept arises, the subject of which is the representation of knowledge - human and computer - in the whole conceptually expressible variety of species, objectified, impersonal, formalized, and personal, implicit, expert knowledge of individuals. Without such a concept, which is called representational work, significant difficulties arise in solving the problems of knowledge engineering. The new epistemological concept of the representation of knowledge becomes the theoretical and methodological basis for integration processes in the field of computer and cognitive sciences that face the problems of combining different concepts and ideas of logic, psychology, philosophy, linguistics and $\mathrm{AI}$ in a single conceptual system.

In the first years of the 21 st century, the transition from the nonclassical stage to the post-nonclassical stage of the evolution of the AI theory begins. A characteristic feature of this stage is the development of the concept of synergistic AI, based, among other things, on quantum-computer technologies. The situation that developed in the AI at the beginning of the new millennium, in a certain sense, resembles the situation of the crisis of classical physics that took place at the turn of the nineteenth and twentieth century's. Classical physics corresponds to the scientific picture of the world, which with minor modifications is given by Cartesian rationalism and Newtonian mechanics. The development of the theory of relativity, quantum mechanics, the quantum-wave theory of light, and a number of other concepts leads in the first decades of the twentieth century to a fundamental break-up of the classical foundation of physics, which later evokes a scientific revolution in physics and a number of related scientific disciplines 
associated with the transition from the classical to the nonclassical type Scientific rationality.

Similar processes are currently taking place in AI. Classical and non-classical AI paradigms and traditional (predominantly logical) models of computer representation of knowledge, whose role in classical AI is similar to Cartesian rationalism and Newtonian mechanics in classical natural science, is gradually becoming secondary to the pressure of new directions, one of the leading places among which begins to occupy the synergetic approach to AI. Proceeding from this, research on the development of general methodological principles of $\mathrm{AI}$, the creation of theoretical foundations of intellectual systems of new generations, development of non-standard hardware and software are becoming topical. Significant prospects in this direction are associated with the application of ideas and synergetic principles in AI.

In recent years, a new post-nonclassical paradigm of AI has been planned, the central core of which will be the concept of synergistic (self-organizing) AI in the near future. Synergetic AI focuses on development scenarios and ways of integrating various intellectual technologies, including the principles and mechanisms of self-organization and the evolution of complex intellectual systems.

There begins to be implemented a cooperative interaction of various means that will allow to compensate for the shortcomings and integrate the advantages of complementary intellectual and other communication technologies [19], and applied to the computer representation of knowledge of various models and languages of representation of knowledge, as a result, will help to obtain synergistic effects.

As is known, the term "synergetic" comes from the Greek word "synergy", meaning conjoint action, cooperation. According to the founder of synergetic G. Haken, this name is successfully suited to the modern theory of self-organizing systems for the following reasons: 1) in the new theory, the conjoint actions of various elements of developing selforganizing systems are investigated; 2) the discovery of the principles of self-organization requires the conjoint efforts of representatives of various scientific directions [20].

The post-classical (synergetic) paradigm of AI even more significantly differs from the classical paradigm than the previously considered non-classical paradigm. The classical and post-non-classical paradigms of AI represent diametrically opposed approaches in AI research. The differences inherent in these two paradigms characterize the methodology, the object of research, the understanding of the nature of the intellect, the interpretation of intellectual systems, the intellect model, the mechanisms for processing information, and, finally, the technologies used.

As a general methodology in the classical paradigm of AI is the traditional rationalistic methodology, whereas in the synergetic paradigm it is replaced by a synergetic methodology. The main object of research in the classical paradigm of AI are simple, closed, static intellectual systems; In the synergetic paradigm of AI, complex, open, dynamic, self-organizing, cooperative intellectual systems become the object of study. The information processing mechanism in the case of the classical AI paradigm can be characterized as strict formal-logical using the basically reliable conclusion; in the post-non-classical paradigm is a combined computational-logic-linguistic mechanism of information processing. The nature of intelligence in the classical paradigm of $\mathrm{AI}$ is understood as an individual; in the synergetic paradigm of AI - as a collective one.

In the classical paradigm of AI, one-level, symbolic models of intelligence are adopted, which are replaced with a synergistic approach to multi-level, symbolic-numerical models. Interpretation of intellectual systems within the framework of the classical AI paradigm deals with onedimensional systems. Synergetic AI interprets intellectual systems as integral, multidimensional, intentional conditioned systems. The classical paradigm of AI uses a classical, homogeneous technology for processing computer data and knowledge. In the synergetic paradigm, heterogeneous, hybrid, cooperative technology is used, which is a synthesis of various intellectual technologies.

The paradigm of synergistic AI includes the study of the processes of formation, activity, communication, evolution and cooperation of intellectual systems. In it, the unstable states, dynamics, mutual transitions, creation and destruction of AI systems are investigated. Examples of synergetic intellectual systems can serve as modern hybrid intelligent systems, systems of so-called soft computing, virtual organizations, multi-agent systems, distributed control systems and a number of others. Finally, it is possible to introduce elements of a synergetic paradigm into classical AI models and methods.

Despite its youth, the theory of AI in half a century of its existence passes in its development of all three types of scientific rationality. The classical type of scientific rationality that dominates natural science for two and a half centuries, from the middle of the 17 th century to the beginning of the 20th century, has lasted less than three decades in AI research - from the mid-1950s to the early 1980s. The non-classical period, which lasted in other sciences for more than half a century - from the beginning of the 1920 s to the end of the 1970 s, has been going on in AI for two decades. Finally, the post-nonclassical type of scientific rationality, which became dominant in most of the sciences by the 1970s, only at the turn of the millennium begins to penetrate the realm of AI.

\section{CONCLUSION}

In the article the concept of the epistemology of artificial intelligence (AI-epistemology) is formulated, which deals with the problems of representation, manipulation, acquisition, replenishment, generalization and classification of computer knowledge, problems of computer perception and computer understanding [21], [22]. The concept of computer epistemology, which explores the problems of understanding the essence of information, is also developed; studying the mechanisms of storage, translation and acquisition of information in computer systems (including computer knowledge); analysis of concepts of knowledge 
engineering. Finally, the concept of computer representation, which analyzes the specifics, formation and development, as well as the most important ways to solve the problem of computer representation of knowledge, is framed in the work.

\section{REFERENCES}

[1] Inozemtsev V.A., Ivlev V.Y., Ivleva M.L., Oseledchik M.B. Representology in the System of Modern Epistemology //Proceedings of the 2016 3rd International Conference on Education, Language, Art and Inter-cultural Communication (ICELAIC 2016). Advances in Social Science, Education and Humanities Research. Paris: Atlantis Press, 2017. Volume 40. P. 697-701.

[2] Luger J. Artificial intelligence: strategies and methods for solving complex problems. M. Williams. 2003.

[3] Barr A., Feigenbaum. The handbook of Artificial Intelligence. Pitman. 1981. V. 1.

[4] Minsky M. Computation and machines. M., Mir. 1971.

[5] Shannon K. Works on information theory and Cybernetics. M., publishing house of foreign literature. 1963.

[6] Pospelov D.A. Logic-linguistic models in management. M., Energoatomizdat. 1981.

[7] Boden M. Artificial Intelligence in psychology. Cambridge (Mass.), MIT Press. 1988.

[8] Oseledchik M.B., Inozemtsev V.A., Ivlev V.Y., Ivleva M.L. LogicalPhilosophical Approach to the Interpretation of the Concept of Knowledge //Proceedings of the 2016 3rd International Conference on Education, Language, Art and Inter-cultural Communication (ICELAIC 2016). Advances in Social Science, Education and Humanities Research. Paris: Atlantis Press, 2017. Volume 40. P.297301.

[9] Ivlev V.Yu., Barkova E.V., Ivleva M.I., Buzskaya O.M. Environmental approach to the study of the modern stage of information society development: research prospects // International Journal of Environmental and Science Education. 2016. V. 11. № 16. pp. 9113-9124.

[10] Rakitov A.I. The Philosophy of the computer revolution. M., Publishing house of political literature. 1991.

[11] Newell A. The knowledge level AI. 1982. V. 18. No. 1. P. 87-127.

[12] Ivlev V.Y., Ivleva M.L., Inozemtsev V.A., Oseledchik M.B. Categories of Modality and Their Use in Modern Biological Conceptions // Proceedings of the 2016 3rd International Conference on Education, Language, Art and Inter-cultural Communication (ICELAIC 2016). Advances in Social Science, Education and Humanities Research. Paris: Atlantis Press, 2017. V. 40. P.687-691.

[13] Philosophy of artificial intelligence. Materials of all Russian interdisciplinary conference. M., IPhRAS. 2005. 399 p.

[14] Dennett D. Kinds of Minds: Towards an Understanding of Consciousness. Basic Books 1997.

[15] Searle J.R. The rediscovery of consciousness. Cambridge (Mass.), MIT Press. 1999.

[16] Winograd T., Florens W. Understanding computers and cognition. N.-Y., Academic Press. 1987.

[17] Stepin V.S. Theoretical knowledge. M., Progress-tradition. 2000.

[18] Inozemtsev V.A. Post-non-classical paradigm in the study of artificial intelligence // Great converters science: M. Planck. Mn., BSUIR. 2006. P. 171-175.

[19] Vasyakin B.S., Ivleva M.I., Pozharskaya E.L., Shcherbakova O.I. A study of the organizational culture at a higher education institution (case study: Plekhanov Russian university of economics (PRUE)) // International Journal of Environmental and Science Education. 2016. V.. 11. № 18. P. 11515-11528.

[20] Haken G. The Mysteries of nature. Synergetic: the doctrine of the interaction. Izhevsk, ICA. 2003.

[21] Chistyakova O. V. Human in a Mediatizing Postmodern World: Anthropological Meanings and Social Implications // Human. Society.
Inclusion. № 2-1 (26), 2016. Published by Moscow State University of Humanities and Economics. pp. 90-98.

[22] Chistyakova O. Philosophical-Anthropological Meanings of Postmodernism: "Mediatizing" Human // Proceedings of the International Conference on Contemporary Education, Social Sciences and Humanities. Advances in Social Science, Education and Humanities Research (ICCESSH 2016). Volume 74. Paris, Atlantis Press, 2016. pp. 637-642 